\title{
FROM COGNITION TO LANGUAGE CHANGE: CLOSE SIMULATION OF A PNG VILLAGE
}

\author{
T. Mark Ellison ${ }^{* 1}$ and Eri Kashima ${ }^{1}$ \\ *Corresponding Author: m.ellison@anu.edu.au \\ ${ }^{1}$ Wellsprings/CoEDL/CHL, Australian National University, Canberra, Australia
}

Research in psycholinguistics and experimental semiotics provide us with new understandings of human communicative processes. Agent-based simulations offer the enticing possibility of converting this understanding into explanations of language change (e.g. Stanford \& Kenny 2013). Such simulations can only be accurate, however, if the interactions between agents pattern like interactions between language community members. In this talk, we present a one-personto-one-agent simulation of a village in southern PNG. To maintain individual anonymity, we will call the village $\mathrm{V}$, and their language $\mathrm{L}$. The opportunities for such simulations are rare, and the only similar study (of which we know) is a model of a small Pacific island community (Hendery \& Magee 2015).

While language $\mathrm{L}$ has closely related languages to its east and west, it has relatively little contact with unrelated languages. It has a small population base - less than a thousand speakers - but does not seem at immediate risk of being supplanted by English, Tok Pisin or Motu.

$\mathrm{V}$ is the smallest of the three villages which identify as L-speaking. It has 136 inhabitants living across 20 households. Life in the village revolves around gardening with the nuclear family, and gender-based activities such as fishing by women. Gardening occurs at hamlets which may be 30 minutes to two hours walk away from the village. These hamlets may be shared between related (or sometimes unrelated) households. During the non-wet seasons between August to December people often stay overnight at the hamlets in shelters built there. During the height of the wet season between January and April people are mostly confined to their houses in the village.

Of the 136 inhabitants in V, 100 are born after 1980. The gender split is 54\% Male to $46 \%$ Female, however the percentage is skewed towards women in the older residents born before 1980 (53\% Female, 47\% Male). Of the 18 married women in the village, 5 have married in from non-L-speaking villages. The small population of the village and the completeness of the data we have on it allow us to build a close simulation of likely interactions.

We simulate language maintenance and innovation in $\mathrm{V}$ by combining de- 
mographic and cognitive modelling. The demographic model combines features of actual persons: domicile proximity, age, first language, gender and gardening area, to determine the interaction likelihood of the corresponding agents. Age also impacts the strength of the egocentric bias (due to weight of past experience). Linguistic change occurs, if at all, as a result of interactions sampled from this likelihood distribution. In each interaction, one randomly selected participant is the speaker, another the hearer.

Tamariz, Ellison, Barr, and Fay (2014) develop a model of selection between variant representations based on earlier experimental semiotics studies. This model assumes that production choices reflect distributions of past experience, modified by two biases. These are the content bias - one variant being inherently more appealing, and an egocentrism bias - the priority agents give to repeating their past representations vs adopting representations which others have used. We adopt this model to account for selection between alternative linguistic forms in $\mathrm{V}$ during interactions.

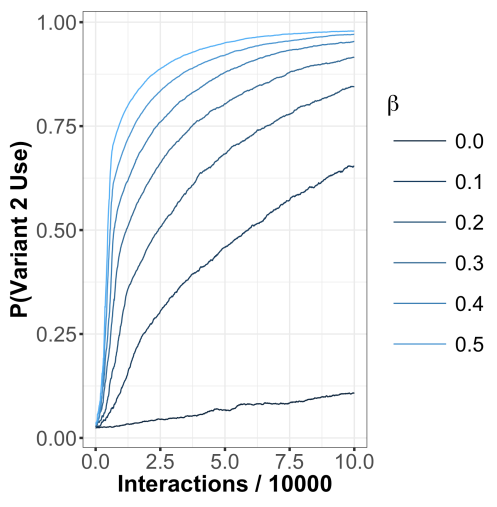

Figure 1. The probability of using introduced variant over time for various levels of content bias $(\beta)$.

achieves some uptake.

We explore tentative evidence for a sound-change in progress in $\mathrm{V}$, and what it can tell us about starting conditions and bias levels. In future work, we will integrate more accurate detailed conditioning of interaction likelihood on inhabitant attributes and daily routines.
One explanation of language change in exogamous communities is that inmarrying women are vectors for new variants; while not the only crosslinguistic contacts in the community, they are arguably the strongest. We explore this option in simulation: with in-married women initialised with two variants at equal likelihood, while everyone else in the village uses only a single variant. People are assumed to be egocentric in their option selection, the strength of this bias to be determined by fit-to-data. Figure 1 explores the impact of varying levels of attractiveness in the new variant. The more attractive the variant, the faster it spreads to the rest of the community. Interestingly, even without a content bias for the new variant, it still 


\section{References}

Hendery, R., \& Magee, L. (2015). Geo-language games: An agent-based model of the role of terrain in language diversity. In Digital humanities 2015. Sydney. Retrieved from http://dh2015.org/abstracts/xml/ HENDERY_Rachel_Marion_Geo_Language_Games_An_Agen /

Stanford, J. N., \& Kenny, L. A. (2013, July). Revisiting transmission and diffusion: An agent-based model of vowel chain shifts across large communities. Language Variation and Change, 25(2), 119-153.

Tamariz, M., Ellison, T. M., Barr, D. J., \& Fay, N. (2014). Cultural selection drives the evolution of human communication systems. Proceedings of the Royal Society B: Biological Sciences, 281(1788), 20140488. 